\title{
Sympathetic and Baroreceptor Reflex Function in Neurally Mediated Syncope Evoked by Tilt
}

\author{
Rogelio Mosqueda-Garcia, ${ }^{* \S}$ Raffaello Furlan,, Roxana Fernandez-Violante, ${ }^{\ddagger}$ Tushar Desai, ${ }^{\star}$ Marie Snell, ${ }^{\star}$ Zoltan Jarai, ${ }^{\star}$ \\ Vasu Ananthram, $\$$ Rose Marie Robertson, ${ }^{\star \S}$ and David Robertson ${ }^{\star} \S$ \\ *Syncope Service in the Autonomic Dysfunction Unit and ${ }^{\ddagger}$ Division of Clinical Pharmacology, ${ }^{\S}$ Department of Medicine, Vanderbilt \\ University Medical Center, Nashville, Tennessee 37232-2195
}

\begin{abstract}
The pathophysiology of neurally mediated syncope is poorly understood. It has been widely assumed that excessive sympathetic activation in a setting of left ventricular hypovolemia stimulates ventricular afferents that trigger hypotension and bradycardia. We tested this hypothesis by determining if excessive sympathetic activation precedes development of neurally mediated syncope, and if this correlates with alterations in baroreflex function. We studied the changes in intraarterial blood pressure (BP), heart rate (HR), central venous pressure (CVP), muscle sympathetic nerve activity (MSNA), and plasma catecholamines evoked by upright tilt in recurrent neurally mediated syncope patients (SYN, $5 \pm 1$ episodes/mo, $n=14$ ), age- and sex-matched controls (CON, $n=23$ ), and in healthy subjects who consistently experienced syncope during tilt $(\mathrm{FS}+, n=20)$. Baroreflex responses were evaluated from changes in HR, BP, and MSNA that were obtained after infusions of phenylephrine and sodium nitroprusside. Compared to CON, patients with SYN had blunted increases in MSNA at low tilt levels, followed by a progressive decrease and ultimately complete disappearance of MSNA with syncope. SYN patients also had attenuation of norepinephrine increases and lower baroreflex slope sensitivity, both during tilt and after pharmacologic testing. FS + subjects had the largest decrease in CVP with tilt and had significant increases in MSNA and heart rate baroreflex slopes. These data challenge the view that excessive generalized sympathetic activation is the precursor of the hemodynamic abnormality underlying recurrent neurally mediated syncope. (J. Clin. Invest. 1997. 99:2736-2744.) Key words: neurocardiogenic syncope - vasovagal • microneurography $\bullet$ tilt $\bullet$ baroreflex
\end{abstract}

\section{Introduction}

Neurally mediated (vasovagal or neurocardiogenic) syncope results from excessive arteriolar dilatation and inappropriate

Part of these results were presented in abstract form at the American Heart Association Annual Scientific Sessions in Atlanta, GA, 8-11 November, 1993 (1993. Circulation [Suppl.]. 88:I-84).

Address correspondence to Dr. Rogelio Mosqueda-Garcia, Clinical Research Center, MCN, Vanderbilt University Medical Center, Nashville, TN 37232-2195. Phone: 615-936-1750; FAX: 615-936-1906; E-mail: Rogelio.Mosqueda@mcmail.Vanderbilt.edu

Received for publication 12 September 1996 and accepted in revised form 13 March 1997.

J. Clin. Invest.

(C) The American Society for Clinical Investigation, Inc. 0021-9738/97/06/2736/09 \$2.00

Volume 99, Number 11, June 1997, 2736-2744 bradycardia (1). In susceptible individuals, this syndrome evolves in response to relative circulating hypovolemia or during emotional overstimulation (2). Syncope resembling that termed "neurally mediated syncope" can also be evoked in otherwise healthy subjects during prolonged upright tilt $(3,4)$, infusion of vasodilator substances (5), or during pronounced lower body negative pressure $(6,7)$. It has been assumed that patients who are prone to this type of syncope have baroreflex mechanisms and hemodynamic responses qualitatively similar to those of healthy subjects and differ only quantitatively $(8,9)$.

Based on experimental observations in nonfainting normal volunteers, it has been proposed that an initial exaggerated sympathetic activation, in combination with relative ventricular hypovolemia, stimulates myocardial ventricular afferents. Stimulation of these afferents may result in reflex vasodilation with or without bradycardia (10-12). Alternatively, others have proposed that a sudden central resetting of baroreflexes produces loss of sympathetic vascular control with subsequent syncope (13).

Some studies have suggested that the vasodilatation seen in neurally mediated syncope results from a withdrawal of sympathetic tone (14-16). Consistent with this view, there is evidence of reduced cardiac and renal norepinephrine (NorEpi) ${ }^{1}$ spillover in patients who fainted during cardiac catheterization (17). In addition, sympathetic withdrawal has been observed using microneurography in healthy subjects undergoing infusion of vasodilator agents $(2,18)$ or during pronounced lower body negative pressure (6). There is only one report studying sympathetic nerve traffic in a patient with a history of vasodepressor attacks (19). In this report, disappearance of muscle sympathetic nerve activity preceded a rather prolonged hypotensive attack, presumably triggered by emotional stress.

To our knowledge, there are no studies in patients with recurrent neurally mediated syncope that characterize the changes in sympathetic nerve activity during the upright posture, the position in which syncope typically occurs (2). Similarly, there is no information that correlates temporal changes in sympathetic nerve activity and NorEpi levels with the development of syncope or with changes in central venous pressure. There is also very little systematic information regarding potential changes in baroreflex function in these patients. In the present studies, in addition to evaluating sympathetic outflow during upright tilt, we studied reflex cardiovascular function in normotensive controls, patients with a medical history of neurally mediated syncope, and healthy subjects who experienced syncope only during tilt.

1. Abbreviations used in this paper: CVP, central venous pressure; Epi, epinephrine; FS + , subjects with false-positive tilt; HR, heart rate; MSNA, muscle sympathetic nerve activity; NorEpi, norepinephrine; SYN, patients with recurrent syncope. 


\section{Methods}

Study groups and patient selection. The subjects abstained from caffeine-containing products and smoking for at least $7 \mathrm{~d}$. All medications were stopped for at least five drug half-lives before the study. The first group of patients included 14 subjects $(5$ males and 9 females, age range $=21-41 \mathrm{yr}$, mean $=34 \pm 1.32 \mathrm{yr}$ ) suffering from recurrent syncope (SYN, two or more episodes per month) who were recruited from the Vanderbilt Autonomic Dysfunction Center. In this group, the diagnosis was established after a review of the patient's medical history, a comprehensive physical examination, and a tilt table study that indicated the presence of this syndrome. In addition, these patients had negative cardiologic (12-lead EKG, echocardiogram, and ambulatory electrocardiographic monitoring), neurologic (electroencephalogram and computed axial tomography assessment), endocrinologic (fasting blood glucose and thyroid function), hematologic, and psychiatric evaluations. The second group (FS+) was comprised of 20 subjects ( 11 males and 9 females, $33 \pm 1.86 \mathrm{yr}$ ) who had no history of syncope and had an unremarkable medical history and physical examination, but nevertheless consistently experienced fainting during tilt. The subjects were identified during the recruitment of normal control subjects for tilt studies. The final group consisted of healthy normotensive controls ( $\mathrm{CON}, n=23)$ who had no syncope or presyncope on tilt. This group included at least one subject who was age- and sex-matched for each patient.

Experimental protocol. The experimental protocol was approved by the Vanderbilt University Institutional Review Board in Human Research, and all the subjects gave written informed consent before any procedure was initiated. An initial $75^{\circ}$ tilt table study was performed (Fig. 1) in which blood pressure was monitored with a Finapress device (Ohmeda, Austell, GA). Patients were excluded if this tilt study was normal. If the study was positive (development of syncope or presyncope resulting from hypotension, bradycardia, or both), the patients were reevaluated 1 wk later with another tilt study. If the response was similar to the first tilt, the patients were included in the remaining portion of the study. A similar approach was used for the inclusion of the control subjects, except that in these individuals, the two tilt tests were negative. The FS + group was formed by subjects with normal medical histories and physical findings, but with consistent positive tilt studies.

For the last part of the study, subjects were admitted to the General Clinical Research Center. After overnight fasting, they underwent another tilt. Blood pressure (BP) was recorded from an intraarterial line placed in the radial artery, and heart rate (HR) was obtained from lead II of the surface electrocardiogram. Central venous pressure (CVP) was obtained using a high fidelity 3-French Millar Mikro-Tip transducer (Millar Instruments, Inc., Houston, TX) placed through the median or basilic vein at the level of the antecubital fossa. In the opposite arm, an intravenous line was positioned for blood sampling or drug administration.

Muscle sympathetic nerve activity (MSNA) was recorded from the right peroneal nerve using the microneurography technique (20, $21)$ described elsewhere $(22,23)$. In brief, the right leg was immobilized and securely positioned on the tilt table using an adjustable leg brace to maximize successful recordings. A unipolar tungsten electrode (uninsulated tip diameter of 1-5 $\mu \mathrm{m}$, shaft diameter of $200 \mu \mathrm{m}$ ) was inserted into the muscle nerve fascicles of the peroneal nerve for multiunit recordings. Nerve activity was fed to a preamplifier $(1,000-$ fold amplification) and filtered using a bandwidth between 700 and $2,000 \mathrm{~Hz}$. The signal was then rectified, amplified $\sim 100$-fold, and integrated (time constant $=0.1 \mathrm{~s}$ ) to obtain a mean voltage display of sympathetic activity. We considered a recording of sympathetic nerve activity acceptable when $(a)$ the microneurography signal demonstrated spontaneous, pulse-coupled bursts of nerve activity that exceeded by threefold the background noise signal; $(b)$ when there was no evidence of increased activity over time; and $(c)$ when activity returned to basal after procedures affecting sympathetic tone. The sympathetic nature of the recordings was indicated by increased activity during the hypotensive phase of the Valsalva maneuver or by inhibition of the activity during the blood pressure overshoot of the same maneuver. From the 26 patients undergoing invasive tilt, MSNA recordings were incomplete, lost, or inadequate in 12 subjects. These are not included in the final analysis. In the remaining 14, the recording electrode did not dislodge, and intact tracings were obtained during the entire tilt, including pre- and postsyncope periods. In five of the completed syncope patients, the recording electrode was subsequently removed to allow leg mobility. The electrode was repositioned later on for performance of the baroreflex study. Data were stored using a digitizing system, and were stored on VCR tape for subsequent analysis.

Tilt procedure. Basal recordings were made $30 \mathrm{~min}$ after instrumentation, including a blood sample for catecholamine determination. Subsequently, the subjects underwent an upright tilt at $15^{\circ}$ intervals every 3 min until reaching $75^{\circ}$. The controls were maintained in this position until 30 min of tilt were completed; the patients and FS+ were placed in supine positions when presyncope or syncope developed. Samples for plasma catecholamines were obtained at the end of each tilt interval and every $5 \mathrm{~min}$ at the maximal tilt angle or, in the corresponding subjects, immediately after syncope or presyncope.

Baroreflex testing. After the tilt, subjects were allowed to rest for the next $45 \mathrm{~min}$ and then, while the subjects were supine, another baseline for hemodynamics and MSNA was recorded for a 3-min period. After this, the subjects received graded infusions of phenylephrine $(0.1-3.2 \mu \mathrm{g} / \mathrm{kg}$ per min) or sodium nitroprusside $(0.1-2.8 \mu \mathrm{g} / \mathrm{kg}$ per min) with the order of the two agents randomized. Infusions were given in ascending order, with each concentration lasting $3 \mathrm{~min}$; the
CONTROLS

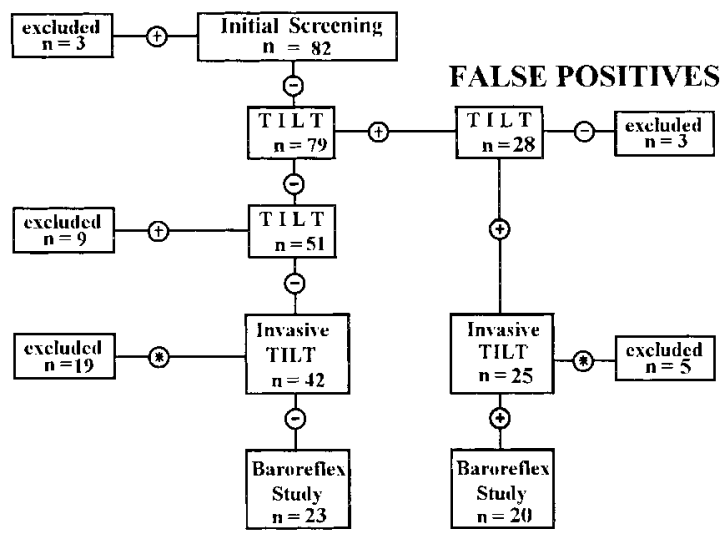

PATIENTS

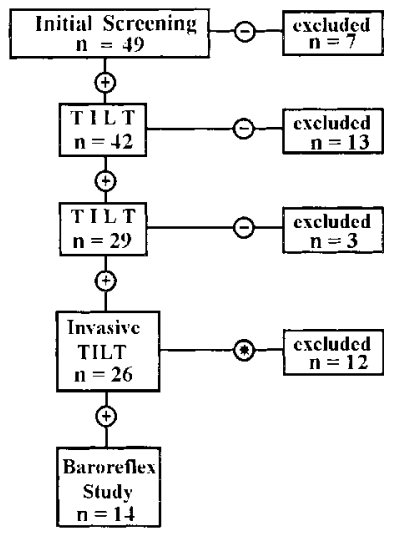

Figure 1. Flowchart of the experimental protocol. A plus sign indicates medical findings compatible with neurally mediated syncope or the development of presyncope or syncope during the tilt table test. A minus sign indicates negative medical findings or a normal tilt table test. An asterisk indicates loss, incomplete, or inadequate recording of muscle sympathetic nerve activity during the tilt. Invasive tilt represents the test done with intraarterial recording of blood pressure, microneurography, and CVP determination. $n$, the number of subjects undergoing each procedure or the number excluded after it. 
concentration was increased until BP rose with phenylephrine by at least 40/30 $\mathrm{mmHg}$ (systolic/diastolic, respectively) or fell with sodium nitroprusside by at least $30 / 20 \mathrm{mmHg}$.

Definitions. We defined neurally mediated syncope as a transient loss of consciousness that was not compatible with other altered states of consciousness in the patient's history. Presyncope was defined as a prodromal period characterized by signs and symptoms of imminent syncope. These included severe lightheadedness preceded by nausea or yawning, severe weakness, transient graying or "tunnel" vision, and/or hearing loss. A positive response to tilt was defined as the development of syncope or presyncope caused by hypotension, bradycardia, or both.

Analyses. For the evaluation of the hemodynamic responses during tilt, we analyzed the entire last minute of each tilt angle and four different 1 -min periods during the $75^{\circ}$ position. Measurements of MSNA were made from the tracings of the microneurogram using a digitizer tablet, which uses computer software for data storage and analysis. Results were evaluated as the number of bursts per minute or as total MSNA (arbitrary units) calculated as bursts per minute times the mean burst amplitude. Since no significant qualitative differences were seen between these two methods of analysis, results are presented as percentage changes in MSNA (bursts per minute) from baseline values. Concentrations of plasma NorEpi and epinephrine (Epi) were obtained using an HPLC method reported previously (24).

Quantification of baroreflex responses to pharmacological manipulations of blood pressure used least squares regression analysis, as reported previously (25). Intervals between each $\mathrm{R}$ wave of the EKG (R-R intervals) and sympathetic nerve activity were averaged during the maximal change in BP. The sigmoid relationship between incremental averages of systolic BP and R-R intervals were then plotted, and the linear portion of these curves was used to derive a slope and a correlation coefficient. A similar process was applied to the relationship between incremental averages of diastolic BP and MSNA.

All data are expressed as mean \pm SEM. Comparisons were analyzed by Student's $t$ test for unpaired observations or ANOVA followed by Dunnett's test for significant differences, as appropriate. $P<0.05$ was considered significant.

\section{Results}

Clinical characteristics. The characteristics of the study groups are presented in Table I. In the patients with recurrent syncope, the duration of symptoms ranged from 7 mo to $10 \mathrm{yr}$, with a frequency of $5 \pm 1.05$ episodes per month. None of the controls or FS+ subjects had contributory medical history or physical findings. Age, weight, diastolic BP, and HR were not significantly different among the groups. Intraarterial systolic BP was lower in subjects with recurrent syncope when compared to the false positive and control groups. Supine plasma NorEpi and Epi values were not different among the study groups (Table I).

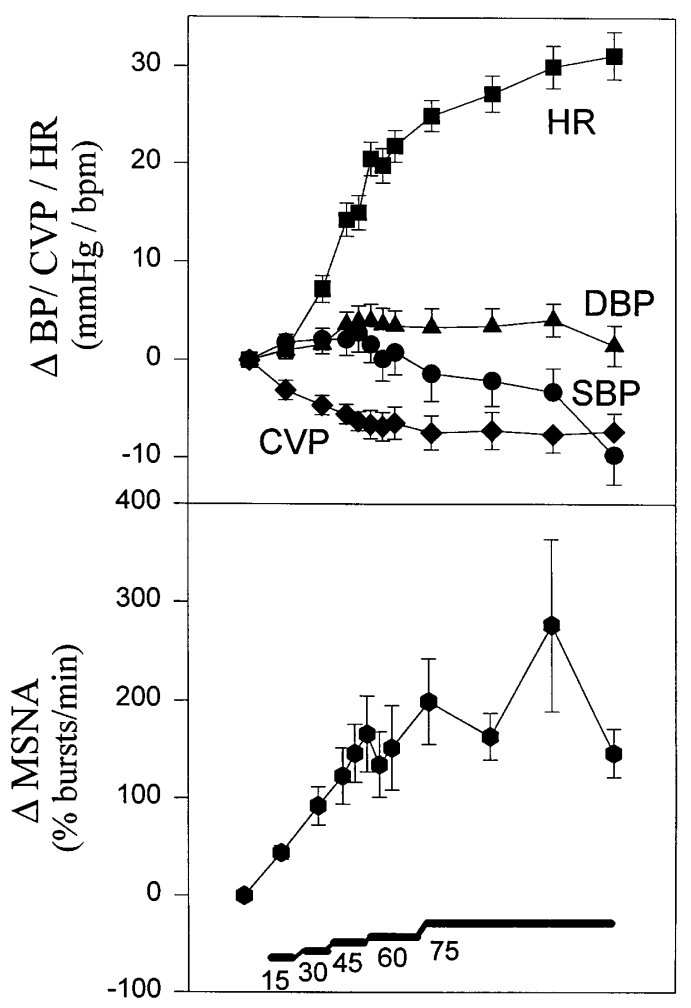

Tilt Angle

Figure 2. Hemodynamic and microneurographic responses to tilt in the control group. The diagram at the top presents the changes in systolic BP (SBP, filled circles), diastolic BP (DBP, filled triangles), HR (filled squares), and CVP (filled diamonds) during different levels of tilt. The diagram at the bottom presents the changes in sympathetic nerve traffic $(\triangle M S N A)$ recorded as percentage change over baseline in bursts $/ \mathrm{min}$. For both diagrams, there is one value for $15^{\circ}$, one for $30^{\circ}$, two for $45^{\circ}$, three for $60^{\circ}$, and four for $75^{\circ}$. The vertical lines represent SEM.

Hemodynamic and microneurographic responses to tilt. The hemodynamic responses to tilt in controls were characterized by a minimal decrease in systolic BP (maximal change at the end of tilt was $-9.68 \pm 3.04 \mathrm{mmHg}$ ), a minor increase in diastolic BP $(4.2 \pm 1.7 \mathrm{mmHg})$, and an initial angle-related decrease in CVP that remained stable after reaching $75^{\circ}$ (maximal decrease of $-7.5 \pm 1.8 \mathrm{mmHg}$; Fig. 2). HR progressively

Table I. Clinical Characteristics of the Study Subjects

\begin{tabular}{ccccccccccc}
\hline & Age & Sex & Weight & SBP & DBP & HR & MSNA & NorEpi & Epi & $n$ \\
\hline & $y r$ & & $k g$ & $m m H g$ & $m m H g$ & $b p m$ & bursts/min & $p g / m l$ & $p g / m l$ & \\
& & & & & & & & & & \\
CON & $33 \pm 1.59$ & $8 \mathrm{M} / 15 \mathrm{~F}$ & $75.87 \pm 1.94$ & $144 \pm 3.68$ & $69 \pm 1.78$ & $65 \pm 1.94$ & $16 \pm 1.6$ & $237 \pm 18$ & $22 \pm 5$ & 23 \\
SYN & $34 \pm 1.32$ & $5 \mathrm{M} / 9 \mathrm{~F}$ & $70.71 \pm 2.89$ & $123 \pm 4.34 *$ & $66 \pm 1.37$ & $68 \pm 3.54$ & $13 \pm 2.73$ & $206 \pm 20$ & $14 \pm 3$ & 14 \\
FS+ & $33 \pm 1.86$ & $11 \mathrm{M} / 9 \mathrm{~F}$ & $69.10 \pm 2.46$ & $139 \pm 4.19$ & $65 \pm 2.19$ & $64 \pm 2.57$ & $16 \pm 1.85$ & $222 \pm 27$ & $60 \pm 34$ & 20
\end{tabular}

CON, control group; SYN, group with recurrent syncope; FS+, subjects with false-positive tilt; SBP, systolic blood pressure; DBP, diastolic blood pressure. *Significantly different from CON $(P<0.001)$ and FS $+(P<0.007)$ groups. 


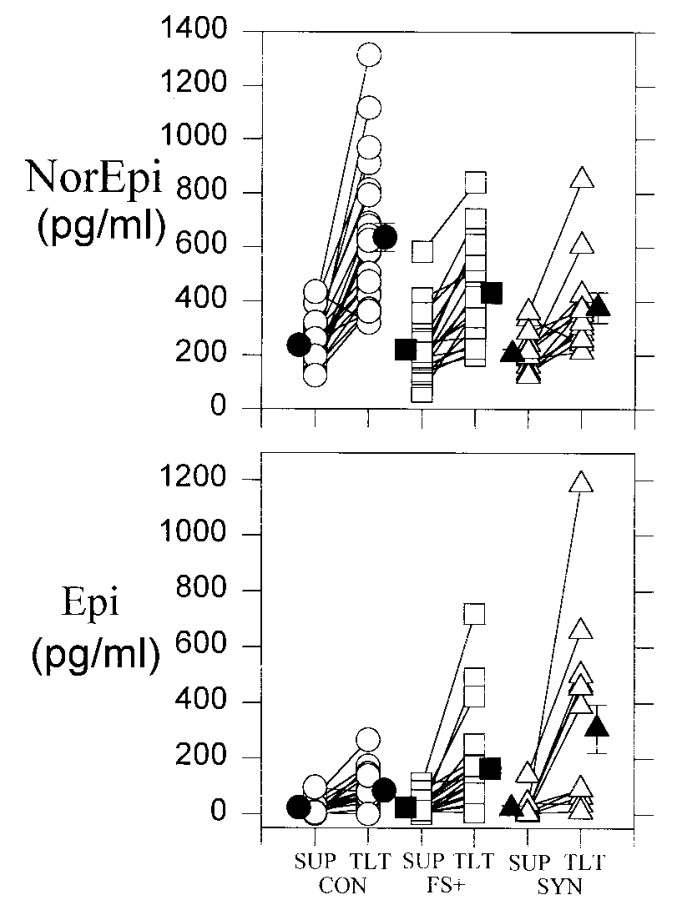

Figure 3. Supine and maximal upright values of plasma NorEpi and Epi during the tilt study. Plasma samples for supine (SUP) NorEpi and Epi were collected in prechilled tubes after the subjects rested at least $30 \mathrm{~min}$ after instrumentation. The maximal values obtained for both catecholamines during tilt (TLT) are presented in this figure. The open symbols represent the individual values for all the participants in the study. The filled symbols at the left and right of the open symbols indicate the mean \pm SEM of each group that was control $(C O N)$, false positive $(F S+)$, and patients with recurrent syncope $(S Y N)$. Note that the maximal NorEpi values were significant lower in the SYN group when compared to CON $(P<0.0003)$ and in the $\mathrm{FS}+$ group when compared to $\mathrm{CON}(P<0.002)$.

increased until reaching a maximal change of $+32 \pm 2.4 \mathrm{bpm}$ at the end of the tilt.

Muscle sympathetic nerve activity in controls increased in response to tilt and, in general, correlated with the degree of tilt angle. The increase in MSNA was maximal within $10 \mathrm{~min}$ at $75^{\circ}(277 \pm 87 \%$ burst $/ \mathrm{min}$, Fig. 2) and remained elevated, although lower than maximum, during the rest of the study. Plasma venous NorEpi and Epi followed a pattern similar to the MSNA with a maximal increase more than threefold over basal values (Fig. 3).

Patients with recurrent syncope developed significant hypotension, even at low levels of tilt (Fig. 4), which progressively worsened and resulted in syncope between $60^{\circ}$ and $75^{\circ}$. The decrease in BP during tilt in these patients was associated with some tachycardia and decreases in CVP that were not significantly different from values observed in controls (compare Figs. 2 and 4). For instance, during the first minute of $45^{\circ}$ tilt, CVP and systolic and diastolic BP had decreased by $-3.2 \pm 0.6,-15.2 \pm 3.4$, and $-6.2 \pm 3.4 \mathrm{mmHg}$, respectively, with a minimal heart rate increase of $10 \pm 3$ bpm (Fig. 4).

More pronounced differences between controls and patients with recurrent syncope were observed when the sympathetic responses to tilt were analyzed. In these patients, the increase in MSNA was severely impaired at low levels of tilt. In

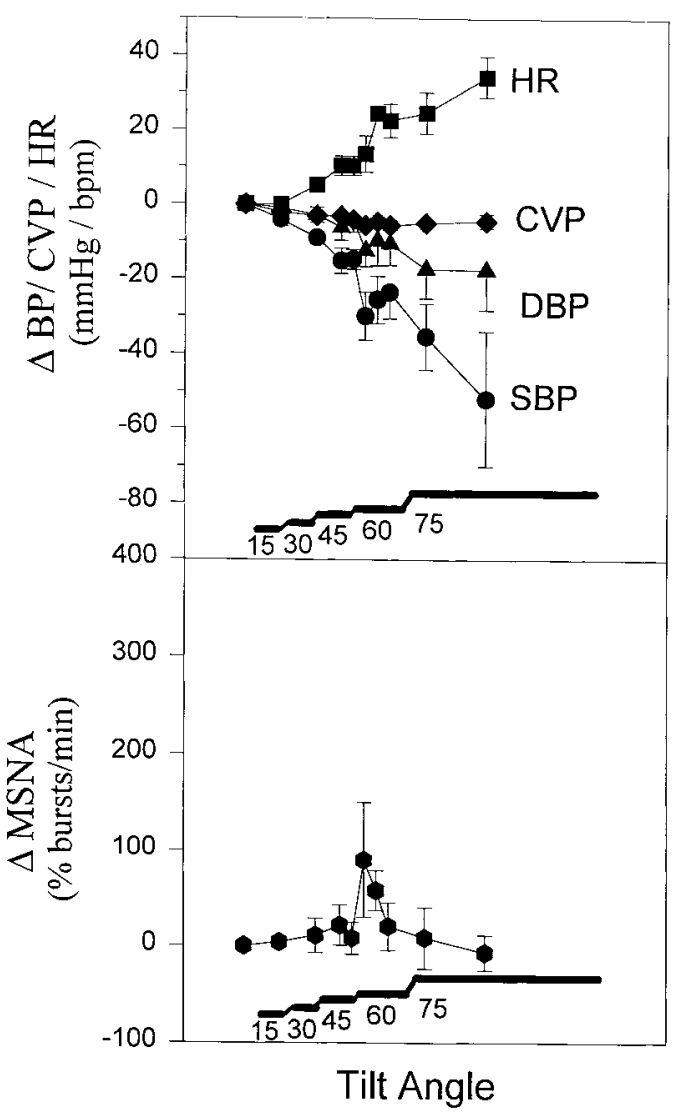

Figure 4. Hemodynamic and microneurographic responses to tilt in patients with recurrent syncope. Arrangement, abbreviations, and symbols are similar to those in Fig. 2. For both diagrams, there is one value for $15^{\circ}$, one for $30^{\circ}$, two for $45^{\circ}$, three for $60^{\circ}$, and two for $75^{\circ}$.

Note that when compared to Fig. 2, there is a different scale for the $y$ axis in the upper tracing and the absence of almost any increase in MSNA during the initial phases of tilt.

addition, the maximal increase in sympathetic activity (which was clearly blunted) was seen earlier, and a phase then followed during which a progressive decrease in MSNA resulted in total neural silence immediately preceding syncope (Figs. 4 and 5). Similar differences were observed when the levels of plasma venous NorEpi were compared between controls and patients. Although supine basal levels were similar, the maximal increase in plasma venous NorEpi in patients $(378 \pm 56 \mathrm{pg} /$ $\mathrm{ml})$ was significantly less than in controls $(637 \pm 52 \mathrm{pg} / \mathrm{ml}, P<$ 0.0003). On the other hand, in the recurrent syncope group, plasma Epi levels were significantly higher than in controls (Fig. 3).

The tilt study in FS + subjects was characterized by a significant decrease in $\mathrm{BP}$ (evident from $30^{\circ}$ ), which resulted in pronounced hypotension and syncope between $60^{\circ}$ and $75^{\circ}$ tilt. In these subjects, the increase in HR reached a maximal change of $25.3 \pm 3.7 \mathrm{bpm}$, and the maximal decrease in CVP was larger than that recorded in the other two groups (Fig. 6). When compared to controls, the changes in MSNA were characterized by similar increments during the low levels of tilt, as well as a tendency for a more pronounced increment at the $60^{\circ}$ and early $75^{\circ}$ tilt. These changes were followed by an abrupt disap- 


\section{TILT ANGLE}

$0^{0}$
$15^{0}$
$30^{0}$
$45^{0}$
$60^{0}$
$75^{0}$

END
CONTROL

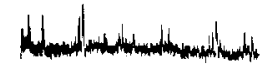

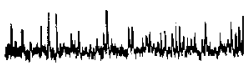
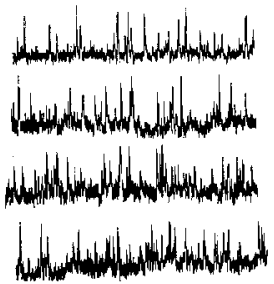

Hith
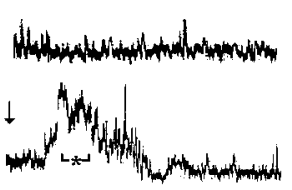

FALSE +
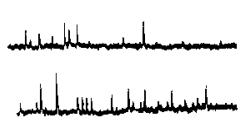

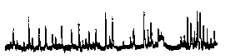
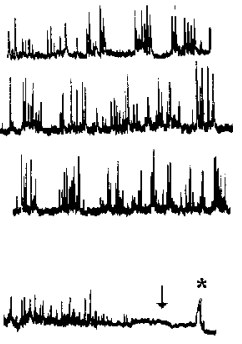

Figure 5. Integrals of microneurographic recordings while supine and during tilt of a control subject, a patient with recurrent syncope (REC. SYNCOPE), and one subject with consistent syncope evoked by tilt $(F A L S E+)$. Recordings were obtained from the right peroneal nerve without moving the recording electrode during the tilt study. Note that in the control subject, the significant increase in MSNA at $15^{\circ}$ and $30^{\circ}$ was followed by a less pronounced increment between $45^{\circ}$ and $75^{\circ}$. In the recurrent syncope patient, there was almost no increase in MSNA at low levels of tilt, and the maximal increment was observed at the first minute of $60^{\circ}$. This was followed by a progressive decrease in MSNA until total disappearance and syncope. The arti-

facts (asterisk) recorded in this patient were produced by involuntary contraction of leg muscles during syncope. In spite of this, note that MSNA reappeared during the recovery period. In the false-positive subject, the maximal increase in MSNA was followed by a more abrupt decrease with total withdrawal and syncope. As with in the middle tracing, the asterisk indicates recording artifacts. The arrows represent the approximate time of syncope.

pearance in MSNA that preceded the development of syncope. Plasma NorEpi and Epi also increased in this group of subjects (Fig. 3). The absolute maximal increase in NorEpi was less than in controls $(P<0.002)$ and similar to the one ob-

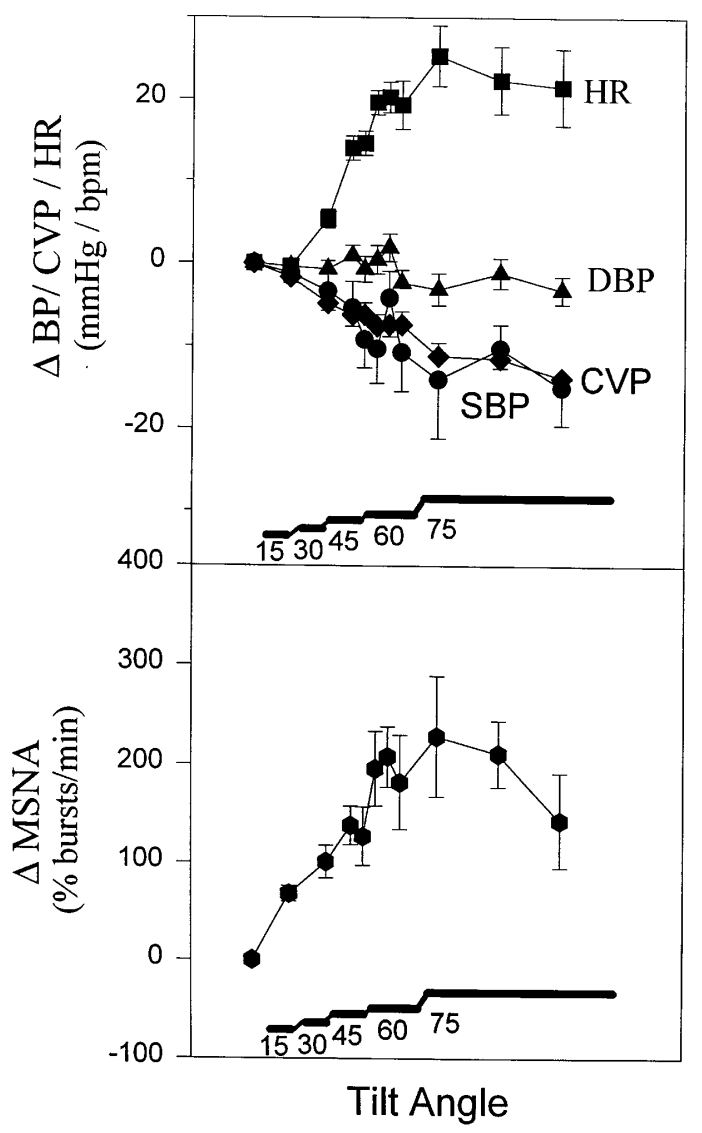

Figure 6. Hemodynamic and microneurographic responses to tilt in false-positive subjects. Arrangement, abbreviations, and symbols are similar to those in Fig. 2. For both diagrams, there is one value for $15^{\circ}$, one for $30^{\circ}$, two for $45^{\circ}$, three for $60^{\circ}$, and three for $75^{\circ}$. Note that when compared to Figs. 2 and 3, there is a different scale for the $y$ axis in the upper tracing, while the scale for MSNA is the same. served in subjects with recurrent syncope, while the increase in plasma Epi was more than in controls $(P<0.025)$.

Reflex cardiovascular responses. Correlation analysis of the hemodynamic changes evoked by tilt ( $\mathrm{R}-\mathrm{R}$ interval versus systolic BP; MSNA versus CVP) indicated significant differences among the three study groups. In controls, the changes between R-R interval and systolic blood pressure yielded a slope of $31.2 \mathrm{~ms} / \mathrm{mmHg}(\mathrm{r}=0.59$; Fig. 7). In the false-positive group, the slope was $43.7 \mathrm{~ms} / \mathrm{mmHg}(\mathrm{r}=0.76)$, while in the group of

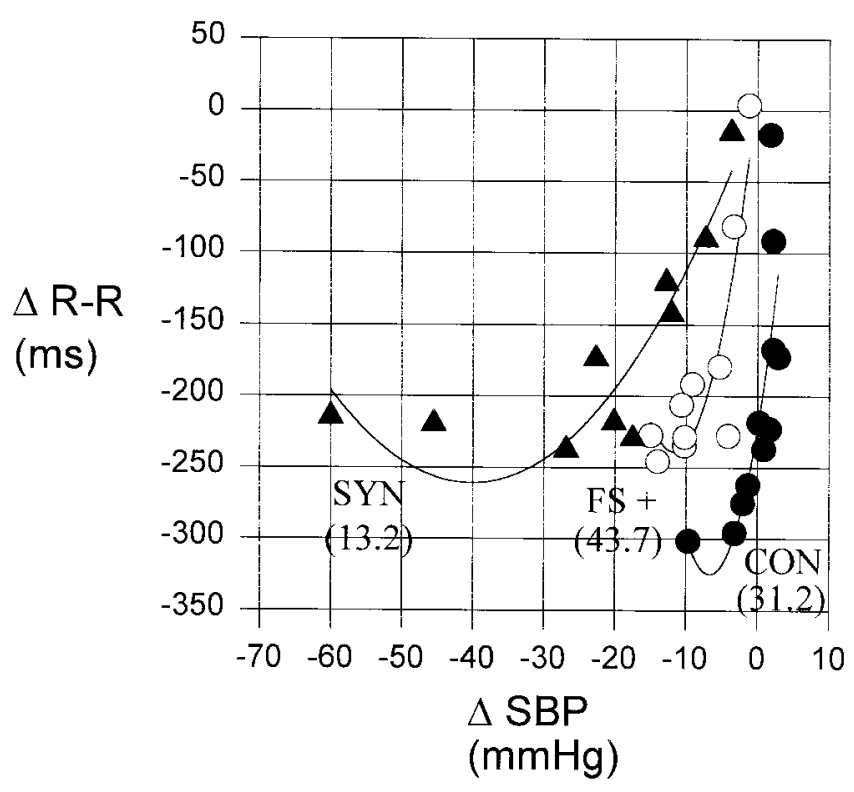

Figure 7. Relationship between changes in R-R interval and systolic $\mathrm{BP}$ during upright tilt. The figure presents the plotted values and regression lines obtained from correlating the changes in R-R interval $(\Delta R-R)$ with the changes in systolic $\mathrm{BP}(\triangle S B P)$ in the three study groups. The filled circles, hollow circles, and filled triangles represent the values obtained in the control subjects $(C O N)$, false-positive subjects $(F S+)$, and recurrent syncope patients $(S Y N)$, respectively. Values between parentheses indicate the regression slope. 


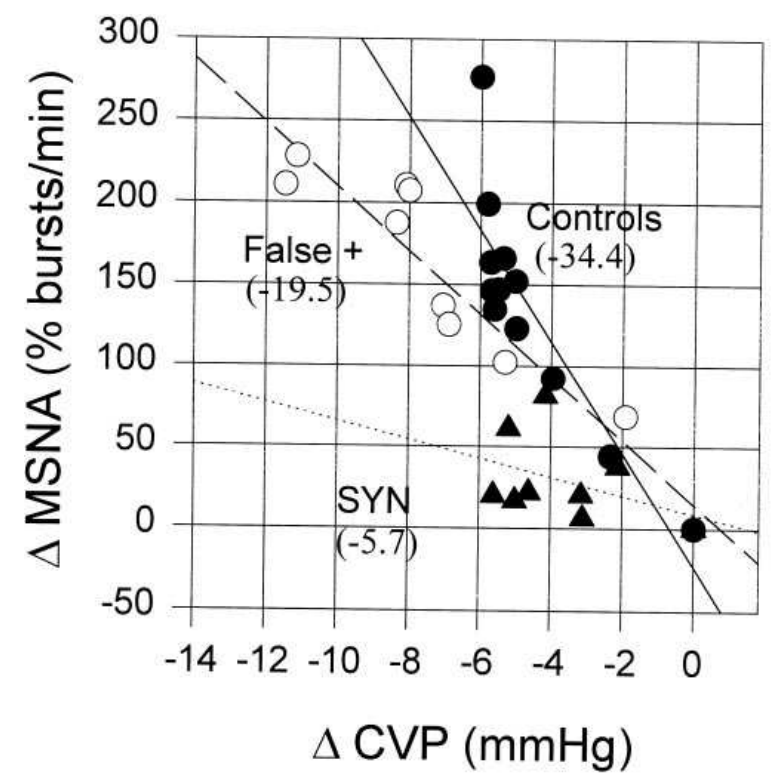

Figure 8. Relationship between changes in muscle sympathetic nerve activity and changes in CVP during upright tilt. The symbols represent the plotted values and regression lines obtained from correlating the changes in MSNA $(\Delta M S N A)$ with changes in CVP $(\Delta C V P)$. Symbols are the same as in Fig. 7.

patients with recurrent syncope, the slope of the curve was significantly reduced $(13.2 \mathrm{~ms} / \mathrm{mmHg} ; \mathrm{r}=0.84$; Fig. 7$)$. The correlation between CVP and MSNA resulted in slope values of -34.4 bursts $/ \mathrm{min}$ per $\mathrm{mmHg}$ for controls $(\mathrm{r}=0.76),-19.5$ bursts/min per $\mathrm{mmHg}$ for the false positive group $(\mathrm{r}=0.90)$, and -5.71 bursts/min per $\mathrm{mmHg}$ for patients with recurrent syncope ( $r=0.15$; Fig. 8$)$.

Using stepwise infusions of phenylephrine and sodium nitroprusside, we further documented alterations in the reflex control of the circulation in subjects with syncope. Among the three groups, patients with recurrent syncope had the lowest baroreflex slopes for both correlations, R-R interval versus systolic BP, and MSNA versus diastolic BP (Fig. 9). No significant differences were observed between controls and falsepositive subjects for the MSNA versus diastolic BP slopes (Fig. 9).

\section{Discussion}

This study reports two major observations relevant to the pathophysiology of neurally mediated syncope. First, there are important and well-defined differences between patients with recurrent syncope and healthy normal subjects who faint only when tilted. Second, significant alterations in the reflex control of the circulation in recurrent syncope patients contribute to the orthostatic intolerance experienced by these subjects.

Our results indicate that the initial sympathetic response to changes in circulating volume is different, not only between controls and patients, but also among different individuals experiencing syncope. In the group with recurrent syncope, the initial increase in MSNA during early tilt was severely reduced. Hemodynamically, these patients had significant decreases in BP that probably resulted from orthostatic reduc-

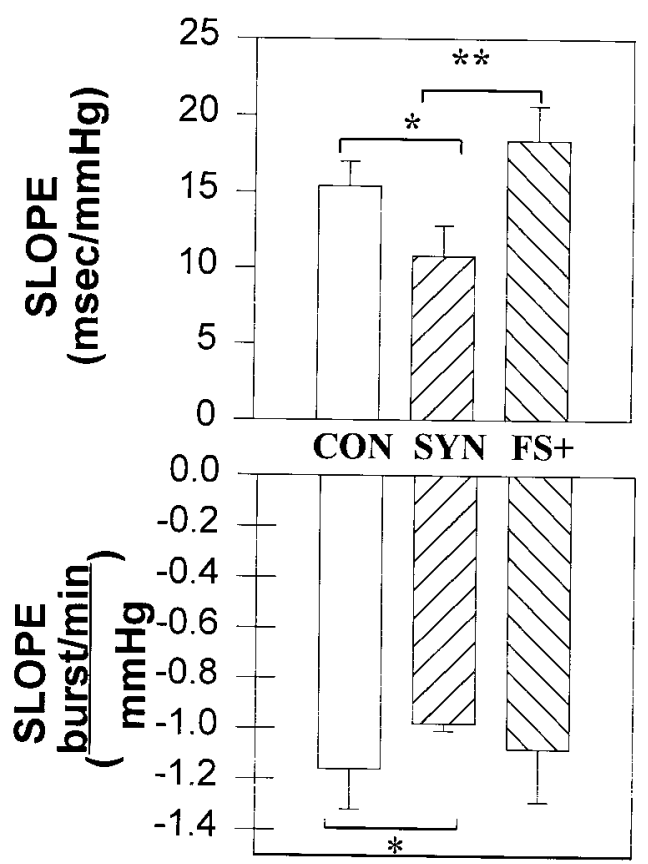

Figure 9. Baroreflex slopes obtained in the study subjects from infusions of phenylephrine and sodium nitroprusside. The bars represent the mean slope value from correlating the changes in R-R interval with systolic BP (slope $\mathrm{ms} / \mathrm{mmHg}$ ) or the changes in MSNA with diastolic BP (slope burst/min per $\mathrm{mmHg}$ ) evoked by ascending infusions of phenylephrine and sodium nitroprusside. The open bars represent the slope values in controls $(C O N)$, while the right ascending hatched and cross-hatched bars represent the slope values for patients with recurrent syncope $(S Y N)$ and false-positive subjects $(F S+)$, respectively. The vertical lines in the middle of each column represent SEM change from baseline. The horizontal lines indicate the difference between the corresponding groups with single and double asterisks representing $P<0.05$ and $P<0.01$, respectively.

tions in circulating volume (as indicated from the decrease in CVP values), with consequent falls in cardiac output and inappropriate reflex tachycardia. In addition, these patients also exhibited reduced maximal increases in MSNA and plasma NorEpi that were inadequate to compensate for the significant fall in arterial BP. This was then followed by progressive MSNA inhibition until complete disappearance and syncope. Attempts to evaluate sympathetic function with plasma NorEpi in neurally mediated syncope have produced variable and seemingly contradictory results; NorEpi concentrations have been reported to be either relatively low $(13,26,27)$, normal (28), or moderately elevated $(4,29,30)$. Confounding factors such as time of sampling, potential changes in NorEpi clearance, and alterations in spillover to the general circulation are likely to account for these contradictory results $(17,31)$. More recently, Jacobs and collaborators have documented a blunted noradrenergic response combined with exaggerated adrenomedullary activation in subjects who subsequently developed vasodepressor reactions during exposure to pronounced lower body negative pressure (13).

The initial MSNA response pattern in patients with recurrent syncope recorded in this study seems to be at variance with earlier published MSNA recordings. It is noteworthy, 
however, that except for one (19), all the microneurographic recordings in previous reports were obtained from subjects without clinical evidence or history of syncope $(6,18,32)$. In the study documenting microneurographic changes in a subject with vasodepressive attacks, the patient had extremely long periods of hypotension (up to $2 \mathrm{~h}$ ) and was studied only while supine. Although this case may represent a form of "central" neurally mediated syncope, in a majority of patients, syncope occurs with upright posture and is of short duration (2). On the other hand, the response pattern in false-positive subjects supports the view that pronounced MSNA increases occur before sudden withdrawal. Together, these observations indicate a heterogeneity in the initial sympathetic response to orthostatic stress in subjects prone to fainting with tilt (but otherwise healthy) and in patients with recurrent syncope. Additional evidence for differential sympathetic responses in neurally mediated syncope is provided by studies assessing spectral analysis of HR variability. These studies have indicated increased $(33,34)$, reduced $(35-37)$, or even nonsustained noradrenergic activation (38) preceding syncope.

Alterations in baroreflex function have been implicit, although not always discussed, when examining the final cardiovascular response of neurally mediated syncope. The concomitant presence of hypotension and inadequate HR response indicate that the normal baroreflex function has been superseded. Some authors have proposed that "sudden" resetting baroreflexes results in sympathoneural withdrawal despite significant hypotension (13). Our results now clearly document that an underlying baroreceptor reflex dysfunction leads to a defective sympathetic response to orthostatic stress in patients with recurrent syncope. In these patients, significant reductions in reflex responses were observed, not only during tilt (changes in CVP versus MSNA and R-R versus systolic BP), but also when baroreflex activity was evaluated with phenylephrine and sodium nitroprusside infusions. The decreased baroreflex function explains the inability of these subjects to increase sympathetic outflow in response to reductions in pressure. Recent reports by at least two different groups of investigators $(39,40)$ have documented similar impairment of baroreceptor reflex sensitivity in different populations of subjects with neurally mediated syncope. In one of these studies, the impairment of baroreflex function predicted the development of syncope during tilt (40). Furthermore, we have recently been able to manipulate the efferent sympathetic limb of the baroreflex in some of these SYN patients (41) and confirm that an enhancement in overall sympathetic tone reduces orthostatic intolerance and prevents syncope induced by tilt. Taken together, these findings support the concept that impairment of baroreflex function is a mechanism that leads to abnormal sympathetic responses to standing in susceptible individuals.

More difficult to clarify is the progressive decrease in MSNA during tilt. One possibility is that in susceptible subjects, the failure to sense changes in pressure may be further complicated by resetting of baroreflexes, which results in sympathetic withdrawal. In laboratory animals, resetting leading to sympathetic inhibition has been described during severe hemorrhage (42) and hypoglycemia (43). In humans, plasma NorEpi first increases and then decreases during progressive reductions of arterial pressure (44). This phenomenon has been ascribed to paradoxical activation of baroreceptors during pronounced hypotension (45). While the degree of inhibition of MSNA de- clines during continuous electrical stimulation of the carotid sinus nerve (46; equivalent to baroreceptor loading) in humans, there has not been clinical experimental evidence demonstrating activation of high pressure baroreceptors at very low arterial pressures.

Implications and limitations of the study. Our findings indicate that in a significant proportion of patients with recurrent neurally mediated syncope, alterations of neural-cardiovascular reflexes result in an inability to adequately respond to orthostatic stress. It may be argued, on the other hand, that a single mechanism (activation of cardiac inhibitory afferents) is responsible for the final sympathetic withdrawal and that the differential changes in sympathetic activity represent only different ends of the spectrum of "normal" response (i.e., subjects in the SYN group had increased sensitivity to sympathetic stimulation of their cardiac afferents while others required pronounced increases). This explanation, however, does not account for the altered baroreflex function and the absence of initial sympathetic activation in response to tilt in several of the present cases. Furthermore, there is emerging evidence arguing against the ventricular theory as the universal mechanism underlying neurally mediated syncope. For instance, using echocardiographic measurements of left cardiac chamber size and determinations of stroke volume, Novak et al. (47) found no evidence of progressive excessive cardiac emptying before the onset of syncope in susceptible patients. More recently, Lui et al. (48) assessed left ventricular wall stress and segmental wall thickening preceding syncope and found no evidence compatible with activation of left ventricular mechanoreceptors.

Assessment of the pathophysiological mechanisms leading to neurally mediated syncope has been difficult, since the spontaneous expressions of this syndrome occur somewhat randomly and usually have resolved by the time that medical evaluation is available. Although the use of tilt table testing has facilitated the study of the hemodynamic characteristics in this type of syncope, questions remain regarding the specificity, sensitivity, and variability of this test (49). In our protocol, we had strict selection criteria that allowed the study of subjects with consistent responses to upright tilt. In their respective groups, subjects had either positive or negative tilt table tests. Individuals in whom the response to tilt was variable (positive/negative or negative/positive) were not studied. Although a remote possibility, this selection process may have excluded subjects with syncope in whom different pathophysiological mechanisms are present. Similarly, our observations relate to posture-induced syncope and might have no relevance to other types of neurally mediated syncope (i.e., the central type, such as emotional fainting).

Finally, the presence of either reduced or increased MSNA in subjects with syncope may also explain the dissimilar therapeutic response observed in different populations $(50,51)$. Conceivably, patients in whom syncope is associated with a blunted increase in sympathetic tone would benefit from $\alpha$-adrenergic receptor agonists or from agents that increase sympathetic tone. In contrast, subjects with exaggerated noradrenergic changes would respond to $\beta$ blockers, while those with pronounced orthostatic decreases in circulatory volume might benefit from mineralocorticoids. Indeed, we believe that our results provide the basis for a more rational design of therapeutic trials in neurally mediated syncope. Our patients with recurrent syncope have responded positively to agents that in- 
crease sympathetic tone, such as direct $\alpha$-adrenergic vascular agonists (midodrine), or to predominantly centrally acting agents (i.e., yohimbine), which increase sympathetic tone, whereas they failed treatment with $\beta$-adrenergic blockers (propranolol, atenolol, etc.). It is therefore reasonable to suggest that the delineation of pathophysiological mechanisms leading to syncope in individual patients will result in better and more rational treatment. This must also be considered when evaluating the efficacy of different pharmacological agents in comparative trials.

In summary, we have found that the sympathetic response in subjects susceptible to neurally mediated syncope is heterogeneous. The finding of altered baroreceptor reflex function in a subpopulation of syncope patients suggests the potential participation of multiple peripheral and/or central mechanisms that eventually lead to severe hypotension and loss of consciousness.

\section{Acknowledgments}

We deeply appreciate the editorial assistance of Mrs. Dorothea Boemer in the preparation of this document.

This work was supported in part by a National Institutes of Health (NIH) Program Project Grant grant HL46681, a grant from the Smokeless Tobacco Research Council, and the Vanderbilt University General Clinical Research Center NIH grant RR00095.

\section{References}

1. Glick, G., and P.N. Yu. 1963. Hemodynamic changes during spontaneous vasovagal reactions. Am. J. Med. 34:42-51.

2. Van Lieshoutt, J.J., W. Wieling, J.M. Karemaker, and D.L. Eckberg. 1991. The vasovagal response. Clin. Sci. 81:575-586.

3. Fitzpatrick, A., G. Theodorakis, P. Vardas, R.A. Kenny, C.M. Travill, A. Ingram, and R. Sutton. 1991. The incidence of malignant vasovagal syndrome in patients with recurrent syncope. Eur. Heart J. 12:389-394.

4. Sander-Jensen, K., N.H. Secher, and A. Astrup. 1986. Hypotension induced by passive head-up tilt: endocrine and circulatory mechanisms. Am.J. Physiol. 251:R742-R748.

5. Robertson, D., G.A. Johnson, R.M. Robertson, A.S. Nies, D.G. Shand, and J.A. Oates. 1979. Comparative assessment of stimuli that release neuronal and adrenomedullary catecholamines in man. Circulation. 59:637-647.

6. Sander, J.S., and D.W. Ferguson. 1989. Profound sympathoinhibition complicating hypovolemia in humans. Ann. Int. Med. 111:439-441.

7. Murray, R.H., L.J. Thompson, J.A. Bowers, and C.D. Albright. 1968. Hemodynamic effects of graded hypovolemia and vasodepressor syncope induced by lower body negative pressure. Am. Heart J. 76:799-811.

8. Blomqvist, C.G, and H.L. Stone. Cardiovascular adjustments to gravitational stress. In Handbook of Physiology. J.T. Shepherd and F.M. Abboud, editors. American Physiological Society, Washington, D.C. 1025-1063.

9. Ludbrook, J. 1989. Faint heart. J. Autonom. Nerv. Sys. 60:83-92.

10. Mark, A.L. 1983. The Bezold-Jarisch reflex revisited: clinical implications of inhibitory reflexes originating in the heart. J. Am. Coll. Cardiol. 1:90102.

11. Abboud, F.M. 1989. Ventricular syncope. Is the heart a sensory organ? New Engl. J. Med. 320:346-351.

12. Waxman, M.B., D.A. Cameron, and R.W. Wald. 1993. Role of ventricular vagal afferents in the vasovagal reaction. J. Am. Coll. Cardiol. 21:1138-1141.

13. Jacobs, M.C., D.S. Goldstein, J.J. Willemsen, P. Smits, T. Thien, R.A. Dionne, and J.W.M. Lenders. 1995. Neurohumoral antecedents of vasodepressor reactions. Eur. J. Clin. Invest. 25:754-761.

14. Barcroft, H., O.G. Edlhom, J. McMichael, and E.P. Sharpey-Schafer. 1944. Posthaemorrhagic fainting. Study by cardiac output and forearm flow. Lancet. i:489-491.

15. Weiss, S., R.W. Wilkins, and F.W. Haynes. 1937. The nature of circulatory collapse induced by sodium nitrite. J. Clin. Invest. 16:73-84.

16. Greenfield, A.D.M. 1966. Survey of the evidence for active neurogenic vasodilation in man. Fed. Proc. Fed. Am. Soc. Exp. Biol. 25:1607-1610.

17. Esler, M., G. Jennings, G. Lambert, I. Meredith, M. Horne, and G. Eisenhofer. 1990. Overflow of catecholamine neurotransmitters to the circulation: source, fate and functions. Physiol. Rev. 70:963-985.

18. Wallin, B.G., and G. Sundolf. 1982. Sympathetic outflow to muscles during vasovagal syncope. J. Autonom. Nerv. Sys. 6:287-291.
19. Yatomi, A., A. Iguchi, K. Uemura, N. Sakamoto, S. Iwase, and T. Mano. 1989. A rare case of recurrent vasodepressive attacks of 2-hour duration: analysis of the mechanism by muscle sympathetic nerve activity recording. Clin. Cardiol. 12:164-168.

20. Vallbo, A.B., K.E. Hagbarth, H.E. Torebjork, and B.G. Wallin. 1979. Somatosensory, proprioceptive and sympathetic activity in human peripheral nerves. Physiol. Rev. 59:919-957.

21. Sundlof, G., and B.G. Wallin. 1978. Human muscle nerve sympathetic activity at rest. Relationship to blood pressure and age. J. Physiol. 274:621-637.

22. Mosqueda-Garcia, R. 1996. Microneurography in neurological research. Am. Acad. Neurol. (Autonom. Nerv. Sys. Sec.). 2:4-5.

23. Davis, S.N., C. Shavers, F. Costa, and R. Mosqueda-Garcia. 1996. Role of cortisol in the pathogenesis of deficient counterregulation after antecedent hypoglycemia in normal humans. J. Clin. Invest. 98:680-691.

24. Rea, R., I. Biaggioni, R.M. Robertson, V. Haile, and D. Robertson. 1990. Reflex control of sympathetic nerve activity in dopamine $\beta$-hydroxylase deficiency. Hypertension (Dallas). 15:107-112.

25. Mosqueda-Garcia, R., C. Tseng, I. Biaggioni, and D. Robertson. 1990 Effects of caffeine on baroreflex activity in man. J. Pharmacol. Exp. Ther. 255: 374-381.

26. Goldstein, D.S., M. Spanarkel, A. Pitterman, R. Toltzis, E. Gratz, S. Epstein, and H.R. Keiser. 1982. Circulatory control mechanisms in vasodepressor syncope. Am. Heart J. 104:1071-1075.

27. Ziegler, M.G., C. Echon, K.D. Wilner, P. Specho, C.R. Lake, and J.A. McCutchen. 1986. Sympathetic nervous withdrawal in the vasodepressor (vasovagal) reaction. J. Autonom. Nerv. Syst. 17:273-278.

28. Sra, J.S., V. Murthy, A. Natale, M.R. Jazayeri, A. Dhala, S. Deshpande, M. Sheth, and M. Akhtar. 1994. Circulatory and catecholamine changes during head-up tilt testing in neurocardiogenic (vasovagal) syncope. Am. J. Cardiol. 73:33-37.

29. Chosy, J.J., and D.T. Graham. 1965. Catecholamines in vasovagal fainting. J. Psychosom. Res. 9:189-194.

30. Fitzpatrick, A., T. Williams, R. Ahmed, S. Lightman, S.R. Bloom, and R. Sutton. 1992. Echocardiographic and endocrine changes during vasovagal syncope induced by prolonged head-up tilt. Eur. J. Cardiac Pacing Electrophys. 2:121-128.

31. Goldstein, D.S. Catecholamines in plasma and cerebrospinal fluid: sources and meanings. In Brain Peptides and Catecholamines in Cardiovascular Regulation. J.P. Buckley and C.M. Ferrario, editors. 15-25.

32. Scherrer, U., S. Vissing, B.J. Morgan, P. Hanson, and R.G. Victor. 1990. Vasovagal syncope after infusion of a vasodilator in a heart-transplant patient. N. Engl. J. Med. 322:602-604.

33. Pruvot, E., J.M. Vesin, J. Schlaepfer, M. Fromer, and L. Kappenberg. 1994. Autonomic imbalance assessed by heart rate variability analysis in vasovagal syncope. Pacing \& Clin. Electrophysiol. 17:2201-2206.

34. Yamamoto, M., H. Sato, S. Abe, T. Namiki, H. Iinuma, L. Fu, T. Aizawa, and K. Kato. 1993. Unusual increase in sympathetic nervous activity preceding syncope in patients with neurally mediated syncope. Circulation. 88(Suppl. I):I-44.

35. Baharav, A., M. Mimouni, T. Lehrman-Sagie, S. Izraeli, and S. Akselrod. 1993. Spectral analysis of heart rate in vasovagal syncope: the autonomic nervous system in vasovagal syncope. Clin. Autonom. Res. 3:261-269.

36. Morillo, C.A., G.J. Klein, D.L. Jones, and R. Yee. 1994. Time and frequency domain analyses of heart rate variability during orthostatic stress in patients with neurally mediated syncope. Am. J. Cardiol. 74:1258-1262.

37. Vardas, P.E., G.E. Kochiadakis, A.E. Orfanakis, S. Chrysostomakis, and E.G. Manios. 1995. Study of the autonomic nervous system in patients with neurocardiogenic (vasovagal) syncope. PACE. 18:845.

38. Lepicovska, V., P. Novak, and R. Nadeau. 1992. Time-frequency dynamics in neurally mediated syncope. Clin. Autonom. Res. 2:317-326.

39. Haruhiko, I., I. Shigeki, M. Mitsuru, U. Tetsuro, M. Naoki, and K. Hiroshi. 1996. Abnormal baroreflex sensitivity in patients with neurally mediated syncope. Circulation. 94(Suppl. I):I-621.

40. Morillo, C.A., K.A. Ellenbogen, M.A. Wood, L.A. Beightol, and D.L. Eckberg. 1996. Impaired cardio-vagal and muscle sympathetic baroreflex outflow in patients with head-up tilt induced neurally mediated syncope. Circulation. 94(Suppl. I):I-544.

41. Mosqueda-Garcia, R., R. Furlan, R. Fernandez-Violante, M. Snell, and D. Robertson. 1996. Enhancement of central noradrenergic outflow prevents neurally mediated syncope. Clin. Autonom. Res. 6:290.

42. Victor, R.G., P. Thoren, D.A. Morgan, and A.L. Mark. 1989. Differential control of adrenal and renal sympathetic nerve activity during hemorrhagic hypotension in rats. Circ. Res. 64:686-694.

43. Nijima, A. 1975. The effect of 2-deoxy-D-glucose and D-glucose on efferent discharge rate of sympathetic nerves. J. Physiol. 251:231-243.

44. Eckberg, D.L., S.W. Harkins, J.M. Fritsch, G.E. Musgrave, and D.F. Gardner. 1986. Baroreflex control of plasma norepinephrine and heart period in healthy subjects and diabetic patients. J. Clin. Invest. 78:366-374.

45. Eckberg, D.L., and P. Sleight. 1992. Afferent baroreceptor activity. In Human Baroreflexes in Health and Disease. D.L. Eckberg and P. Sleight, editors. Oxford University Press, New York. 123-152.

46. Wallin, B.G., G. Sundlof, and W. Delius. 1975. The effect of carotid si- 
nus nerve stimulation on muscle and skin nerve sympathetic activity in man. Pflugers Archiv. Eur. J. Physiol. 358:101-110.

47. Novak, V., G. Honos, and R. Schondorf. 1996. Is the heart empty at syncope? J. Autonom. Nerv. Sys. 60:83-92.

48. Liu, J.E., R.T. Hahn, K.M. Stein, S.M. Markowitz, P.M. Okin, R.B. Devereux, and B.B. Lerman. 1996. Does mechanoreceptor stimulation explain neurally mediated syncope? Circulation. 94(Suppl. I):I-334.

49. Kapoor, W.N., M.A. Smith, and N.L. Miller. 1994. Upright tilt testing in evaluating syncope: a comprehensive literature review. Am. J. Med. 97:78-88. 50. Kaufmann, H. 1995. Neurally mediated syncope: pathogenesis, diagnosis, and treatment. Neurology. 45(Suppl. 5):s12-s18.

51. Sra, J.S., M.R. Jazayeri, B. Avitall, A. Dhala, S. Deshpande, Z. Blanck, and M. Akhtar. 1993. Comparison of cardiac pacing with drug therapy in the treatment of neurocardiogenic (vasovagal) syncope with bradycardia or asystole. N. Engl. J. Med. 328:1085-1090. 